\title{
The Genetics Corner: HNF1B-Related Bilateral Cystic Kidney Disease in an Infant and his Asymptomatic Mother
}

Robin Dawn Clark MD

\section{Case History:}

A 5-year old boy with end-stage renal disease presented for a genetic evaluation with his foster mother.

Bilateral cystic kidneys were identified prenatally at the 20-week fetal anatomy ultrasound scan in an otherwise uncomplicated pregnancy. An amniocentesis was offered and declined. Parental renal ultrasound exams were recommended. Polycystic kidneys were identified in the asymptomatic and healthy 22-year old G2P1 mother. A male infant was born at 34 weeks gestation by emergency $\mathrm{C}$-section. He was in the NICU at another facility for a month and then institutionalized at a chronic care facility for his first three years of life. At age 3 , he was discharged to a foster family. At age 5, when he presented to us for genetic evaluation, he had chronic kidney disease, stage $\mathrm{V}$, treated with nightly peritoneal dialysis, and he was fed by gastrostomy tube. He was being evaluated for diabetes mellitus due to high glucose levels.

\section{"At age 5, when he presented to us for genetic evaluation, he had chronic kidney disease, stage $V$, treated with nightly peritoneal dialysis, and he was fed by gastrostomy tube. He was being evaluated for diabetes mellitus due to high glucose levels."}

Parents are of African American ancestry. Consanguinity was denied. In addition to his biological mother, several other maternal family members had chronic renal disease, which followed an autosomal dominant pattern of inheritance. A maternal uncle in his early 30s had a polycystic kidney disorder, but he did not require dialysis. The maternal grandfather had polycystic kidney disease that required dialysis. He died at age 63 of pancreatic cancer. A maternal great uncle had renal failure that required dialysis.

The physical exam was pertinent for poor growth: weight: 18.9 $\mathrm{kg}, 48 \%$ ile, $\mathrm{Z}=-0.06$, height: $111 \mathrm{~cm}, 52 \%$ ile, $Z=0.05$, head circumference: $49 \mathrm{~cm}, 3.92 \%$ ile, $Z=-1.76$. No significant dysmorphic features were noted.

Echocardiogram, bone age, and chromosome microarray were normal. Laboratory studies revealed an elevated hemoglobin $\mathrm{A} 1_{\mathrm{c}}$, 6.4 (reference range: 4.8-5.95). A gene panel was ordered (Autosomal Dominant Polycystic Kidney Disease Panel: PKD1, PKD2, PKHD1, HNF1B, DNAJB11, DZIP1L, GANAB) that identified a pathogenic heterozygous variant in $H N F 1 B$ that led to a prema- turely truncated protein: c.826C>T, p.Arg276*. This established his diagnosis as autosomal dominant $H N F 1 B$-related renal disease at age 5 .

\section{Discussion:}

\section{Background on CAKUT}

The prevalence of congenital anomalies of the kidney and urinary tract, referred to as CAKUT, ranges from 3-6 per 1,000 births. This heterogeneous group of anomalies includes a wide range of conditions: renal agenesis, hypodysplasia, multicystic dysplastic kidney, horseshoe or ectopic kidney, anomalies of the collecting system (ureteropelvic junction obstruction, posterior urethral valve, hydroureter, duplicated collecting system) and bladder (vesicoureteral reflux, ureterocele). CAKUT is the most common cause of end-stage renal disease in childhood, accounting for over $40 \%$ of all children who require renal replacement therapy. More than two-thirds of CAKUT patients progress to ESRD as adults.

\section{"CAKUT is the most common cause of end-stage renal disease in childhood, accounting for over $40 \%$ of all children who require renal replacement therapy. More than two-thirds of CAKUT patients progress to ESRD as adults. "}

\section{Is a prenatal diagnosis of CAKUT reliable?}

Although in our patient, the prenatal diagnosis of bilateral renal cystic disease was confirmed postnatally, that is not always the case. A prenatal ultrasound examination is not as reliable as a postnatal ultrasound for the diagnosis of CAKUT. Prenatal US exams produce both false negative and false positive CAKUT diagnoses. The positive predictive value and sensitivity of prenatal US for the detection of CAKUT can be calculated from data published by Li et al. (2019), who determined the true incidence of CAKUT in a series of neonates who were screened with both prenatal and postnatal ultrasonography (US). Although this work was published in Chinese, an English-language abstract provides enough data to perform these calculations. The authors found CAKUT on postnatal US in $3.1 \%$ of their study group (82/2655). Although 44 neonates in the sample were prenatally diagnosed with CAKUT, only 35 of these were true positives (TP), with a confirmed postnatal diagnosis of CAKUT. The predictive value of a positive prenatal US diagnosis of CAKUT in this sample was 79.5\% (TP/TP+FP; 35/44).

Further, among the 2611 neonates who had a normal prenatal 
ultrasound exam, $1.8 \%$ had a CAKUT on the postnatal US, yielding 47 infants with CAKUT after a falsely negative, normal prenatal US (FN; false negatives; 47). These false negatives represent more than half of their total of 82 confirmed CAKUT cases. In this study, the sensitivity of prenatal US for detecting CAKUT was $42.7 \%$ (TP/TP+FN; 35/35+47). This data suggest that all prenatal CAKUT diagnoses should be confirmed with postnatal imaging and that a normal prenatal US does not rule out a renal anomaly.

\section{Should we screen asymptomatic first-degree relatives for CAKUT?}

CAKUT is often familial, even when the family history does not reflect it. The frequency of familial CAKUT ranges from $7-50 \%$. Bulum et al. (2013) studied the family histories of 218 index patients with CAKUT in Ankara, Turkey, and identified 72 affected individuals in 50 families $(22.9 \%)$ who were known to have a CAKUT. These authors also performed ultrasound exams in 504 asymptomatic first-degree relatives of 180 of their index patients with CAKUT and identified a CAKUT in 116 (23\%) individuals from 87 families (48.3\%). When both family histories and ultrasound results in asymptomatic first-degree relatives were considered together, 129/504 first-degree relatives (25.6\%) in 92/180 families $(51.1 \%)$ had a CAKUT. Consanguinity was reported in $24.8 \%$ of these index patients. The rate of autosomal recessive traits would be expected to be higher in the consanguineous families, but two affected children were born to unaffected parents in only ten families $(5.6 \%)$.

In India, Manoharan et al. (2020) identified a familial clustering of CAKUT among $7.9 \%$ of 270 asymptomatic first-degree relatives of 138 index cases. In another study, from Istanbul, Turkey, Gok et al. (2020) found familial CAKUT in 14.4\% (21/145 families) of 412 asymptomatic first-degree relatives of 145 index patients with CAKUT. The risk for familial CAKUT was higher among patients with renal agenesis. Among 5 index cases with renal agenesis, 3 had an affected asymptomatic first-degree relative. The type of anomaly was comparable between the index cases and affected first-degree relatives in 11/21 CAKUT families (51\%). In 6 of the 21 affected families, CAKUT was diagnosed only in siblings, suggesting an autosomal recessive disorder. Familial CAKUT was present in $13.8 \%$ of the consanguineous families (4/29) and $14.6 \%$ of the non-consanguineous families (17/116). In this study, consanguinity contributed little to the risk of familial CAKUT, probably because of the predominance of autosomal dominant disorders.

These and other studies suggest that US screening of asymptomatic first-degree relatives of individuals with CAKUT may be reasonable. However, there are no official guidelines on screening family members because the cost-effectiveness of this strategy has not been established. Such a cost-benefit analysis should determine the relative efficacy of family screening based on the type of anomaly and for unilateral vs. bilateral CAKUT. We know that genetic testing has a higher yield in patients with bilateral CAKUT, and it is reasonable to wonder whether familial screening might be more cost-effective in these patients as well.

\section{What are the features of HNF1B-associated Renal Cysts and Diabetes syndrome?}

Although many genes contribute to the risk for familial CAKUT, variants in the hepatocyte nuclear factor 1 beta, $H N F 1 B$, are the most common monogenic cause of developmental renal disease, responsible for $5-15 \%$ of cases. Both pathogenic variants in gene sequence and large deletions of chromosome 17q12 that include this gene can cause Renal Cysts and Diabetes (RCAD) syndrome, also referred to as Maturity Onset Diabetes of the Young, Type 5 (MODY5; OMIM 137920). The renal disease in RCAD syndrome is highly variable and ranges from a small or single kidney, horseshoe kidney, hyperuricemic nephropathy to renal cysts. Of these, renal cysts were the most common, present in 19/23 patients, in the series reported by Edghill et al. (2006). Eleven of 23 patients in this cohort had diabetes, which is typically diagnosed before age 25 years. Two $(9 \%)$ had genital tract malformations: uterus didelphys with a single ovary and hemi-uterus, respectively, whereas another female mutation carrier had unexplained primary infertility. The HNF1B-associated disease is a multisystem disease that can include pancreatic hypoplasia, genital tract malformations (vaginal aplasia, rudimentary uterus), early-onset gout, abnormal liver function, and hypomagnesemia. Autism and cognitive impairment may occur in individuals with $17 q 12$ deletion. Our patient has early evidence of hyperglycemia, suggesting that he may be developing diabetes associated with this disorder in childhood.

\section{Do bilateral CAKUT increase the yield of genetic testing?}

Bilateral renal anomalies increase the likelihood of finding a gene mutation. Ishiwa et al. (2019) performed a retrospective analysis of 66 patients with CAKUT who underwent gene analysis. Of these, $21.2 \%(14 / 66)$ had detectable pathogenic variants in HNF1B (7/14), PAX2 (Renal coloboma syndrome, 4/14), EYA1 (Branchio-oto-renal syndrome, 1/14) CHD7 (CHARGE syndrome, 1/14) and EP300 (Rubinstein-Taybi syndrome, 1/14). Bilateral renal lesions were significantly more likely to have a detectable gene variant, $32.5 \%$ (13/40; $p=0.02)$. An HFN1B variant was identified in $7 / 14$ patients, all of whom had bilateral hypodysplastic, polycystic or multicystic kidneys. Of these, 2/7 had point mutations in $H N F 1 B$, and $5 / 7$ had $17 q 12$ deletion syndrome, illustrating the value of chromosome microarray in the evaluation of CAKUT.

Patients with CAKUT who are diagnosed in the newborn period are in a unique position to have an early genetic diagnosis, which allows their medical providers to anticipate complications and better manage their disease and to provide genetic counseling and testing for at-risk relatives. Our patient did not have genetic testing during the newborn period. This missed opportunity delayed his diagnosis for 5 years.

\section{Practical Applications:}

1. When an infant has a CAKUT, take a detailed family history for renal disease, diabetes, and other anomalies

2. Consider renal US screening in asymptomatic first-degree relatives of infants with CAKUT, especially the baby bilateral involvement with at least one multicystic or polycystic kidney.

3. Search for other anomalies (eye, ear, cardiac, limb, genital) to determine if CAKUT is isolated or syndromic and choose genetic tests accordingly.

4. Order genetic testing during the newborn period whenever possible and especially when CAKUT is bilateral. Order chromosome microarray (for 17q12 deletion). When CAKUT 
is apparently isolated or nonsyndromic but bilateral, choose a gene panel that includes $H N F 1 B$

References:

1. Bulum B, Ozcakar ZB, Dusunceli, et al. High frequency of kidney and urinary tract anomalies in asymptomatic firstdegree relatives of patients with CAKUT. Pediatr Nephrol. 2013;28:2143-2147.

2. Edghill EL, Bingham C, Ellard S, et al. Mutations in hepatocyte nuclear factor-1-beta and their related phenotypes. $J$ Med. Genet 43: 84-90, 2006. PubMed: 15930087,

3. Gök ES, Ayvacı A, Ağbaş A, et al. The frequency of familial congenital anomalies of the kidney and urinary tract: Should we screen asymptomatic first-degree relatives using urinary tract ultrasonography? Nephron. 2020;144(4):170-175. PMID: 31910412

4. Ishiwa S, Sato M, Morisada N, et al. Association between the clinical presentation of congenital anomalies of the kidney and urinary tract (CAKUT) and gene mutations: an analysis of 66 patients at a single institution. Pediatr Nephrol. 2019;34(8):1457-1464. PMID 30937553

5. Li N, Ji L, Chao S, et al. Ultrasound screening and follow-up study of congenital anomalies of the kidney and urinary tract in neonates. Journal of Peking University (Health Sciences), 2019, 51(6):1062-1066. PMID 31848505

6. Manoharan $A$, Krishnamurthy $S$, Sivamurukan $P$, et al. screening for renal and urinary tract anomalies in asymptomatic first degree relatives of children with congenital anomalies of the kidney and urinary tract (CAKUT) [published online ahead of print, 2020 Mar 20]. Indian J Pediatr. 2020;10.1007/s12098-020-03262-7. PMID 32198693

The author has no relevant disclosures.

NT

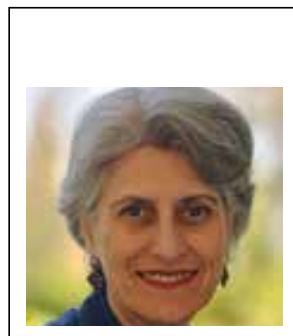

Robin Clark, MD

Professor, Pediatrics

Loma Linda University School of Medicine

Division of Genetics Department of Pediatrics

rclark@llu.edu

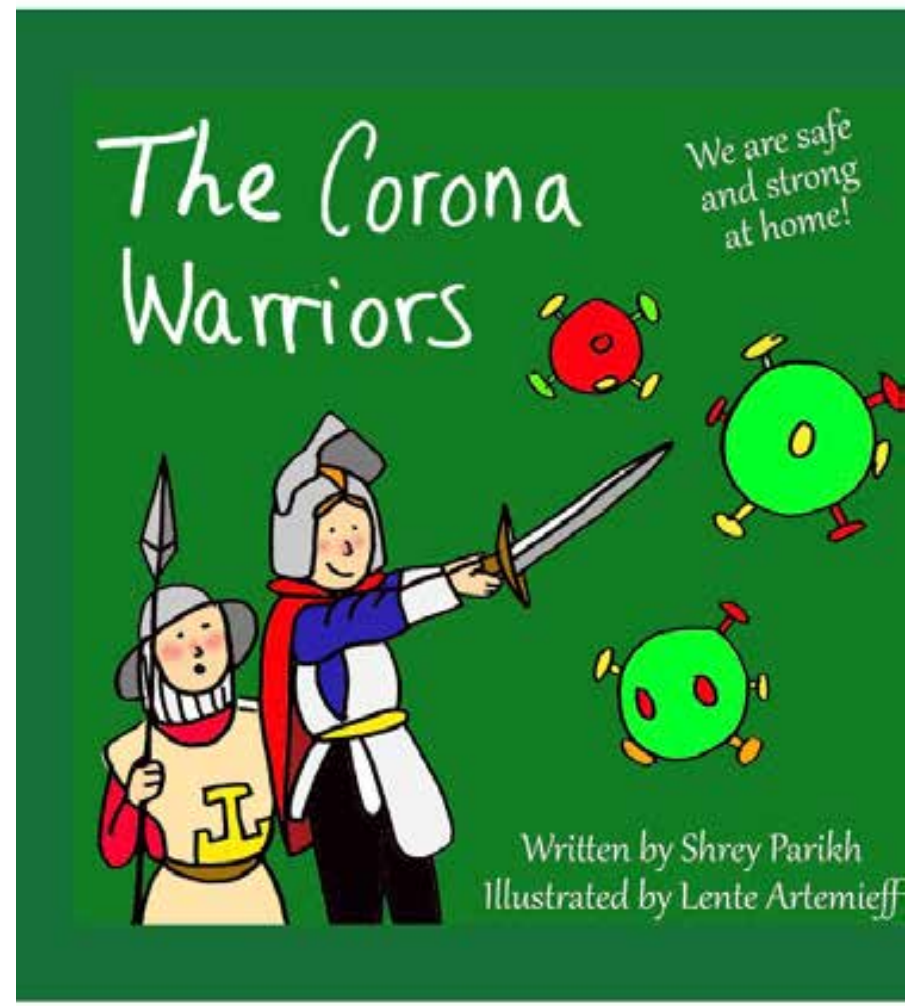

Corresponding Author

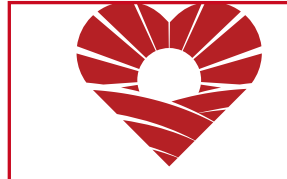

CONGENITAL CARDIOLOGY TODAY
The only worldwide monthly publication exclusively serving Pediatric and Adult Cardiologists that focus on Congenital/ Structural Heart Disease (CHD), and Cardiothoracic Surgeons. 